The val i di ty of mul ti-cent er common nor mal dat abase for i dent i fyi ng myocar di al i schema: Japanese Soci ety of Nucl ear Medi ci ne Wbrking Group Dat abase

\begin{tabular}{|l|l|}
\hline 著者 & $\begin{array}{l}\text { Nakaj i ma Keni chi, Nat suo Shi nr o, Kawano } \\
\text { Nasaya, Mat sunto o Naoya, Hashi not o Jun, } \\
\text { Yoshi naga Kei i chi ro, Taki Juni chi, Okuda } \\
\text { Koi chi }\end{array}$ \\
\hline $\begin{array}{l}\text { j our nal or } \\
\text { publ i cat i on t i t l e }\end{array}$ & Annal s of Nucl ear Nedi ci ne \\
\hline vol une & 24 \\
\hline nunber & 2 \\
\hline page r ange & $99-105$ \\
\hline year & $2010-02-01$ \\
\hline URL & ht t p: //hdl . handl e. net /2297/23923 \\
\hline
\end{tabular}




\title{
The validity of multi-center common normal database for identifying myocardial ischemia: Japanese Society of Nuclear Medicine working group database
}

Kenichi Nakajima (1), Shinro Matsuo (1), Masaya Kawano (2), Naoya Matsumoto (3), Jun Hashimoto (4), Keiichiro Yoshinaga (5), Junichi Taki (1), Koichi Okuda (6)

(1) Department of Nuclear Medicine, Kanazawa University Hospital, Kanazawa, Japan

(2) Department of Radiology, Kanazawa Cardiovascular Hospital, Kanazawa, Japan

(3) Department of Cardiology, Nihon University Surugadai Hospital, Tokyo, Japan

(4) Department of Radiology, Keio University, Tokyo, Japan (Current: Department of Radiology, Tokai University School of Medicine, Isehara, Japan)

(5) Department of Molecular Imaging, Hokkaido University Graduate School of Medicine, Sapporo, Japan

(6) Department of Biotracer Medicine, Kanazawa University, Kanazawa, Japan

Correspondence:

Kenichi Nakajima, MD, Department of Nuclear Medicine, Kanazawa University Hospital

13-1 Takara-machi, Kanazawa, 920-8641, E-mail: nakajima@med.kanazawa-u.ac.jp

Tel 076-265-2333, Fax 076-234-4257

\begin{abstract}
Purpose. The Japanese Society of Nuclear Medicine (JSNM) working group has created a myocardial perfusion imaging database applicable to standard acquisition protocol. The aim of this study is to validate the diagnostic accuracy of the common normal database compared with the expert interpretation of each institute.

Methods. Five institutions participated in this study and used different acquisition settings which included $360^{\circ} / 180^{\circ}$ rotation, camera configuration and camera orbits. The software and its version used in each institution also varied. The working group database was applied to detect the culprit coronary territory from a total of 166 patients with coronary artery disease (CAD) and 145 patients with low-likelihood of CAD.

Results. When summed stress score $\geq 4$ was defined as significant abnormality, overall sensitivity, specificity and accuracy of patient-based analysis were $77 \%, 72 \%$ and $75 \%$, respectively, based on quantitative analysis using the common database, whereas those by institutional visual expert reading were $72 \%, 79 \%$ and $75 \%$, respectively.

Conclusion. The common database, which was created by a multi-center working group and separated between male/female with $180 / 360^{\circ}$ acquisitions, demonstrated comparable diagnostic accuracy to expert interpretation by each institute, and it may be applicable to multi-center studies.
\end{abstract}

\section{Key Words:}

myocardial perfusion imaging; normal database; Japanese Society of Nuclear Medicine working group; coronary artery disease; quantification 


\section{Introduction}

Myocardial perfusion imaging has been applied to detect myocardial ischemia, therapeutic decision making and prognostic estimates, and is a well-established diagnostic tool. In the preceding studies, although visual evaluation of extent and severity of ischemia was essential, quantitative analysis aided or enhanced a diagnostic accuracy [1-3]. Since appropriate normal databases matching those of the acquisition parameters, genders and types of tracers were considered indispensable, we accumulated normal databases from multiple institutions as the working group activity of the Japanese Society of Nuclear Medicine (JSNM) [4-6]. Our aim was to use one common standard database for multiple centers with nuclear medicine facilities. However, whether it was applicable to multiple centers and was comparable to expert interpretation of each institution were not clarified. Therefore we hypothesized that the common database sets could be applied to different combinations of camera and software types. The results will also help with better understanding of the quantitative results from different institutions based on uniform or standard diagnostic criteria.

\section{Methods}

\section{JSNM 2007 database}

The JSNM working group database for myocardial perfusion tracers was created in 2007, and the characteristics of the database, backgrounds of a low-likelihood of coronary artery diseases, type of tracers and acquisition conditions were described elsewhere [4]. Briefly, the perfusion databases consisted of 326 sets of short-axis images, and they were classified into male/female, $180^{\circ} / 360^{\circ}$ and tracer type combinations. The data were collected from nine nuclear medicine centers, where standard acquisition protocols with multi-detector single-photon emission computed tomography (SPECT) were used. All the institutions used a gated SPECT study, but they did not use attenuation correction methods. The accumulation of data was approved by the ethical committee of each respective institution.

The creation and distribution of the databases in the present study were schematically shown in Figure 1. The normal database files were created at Kanazawa University for QGS (Cedars Sinai Medical Center, USA) with different versions and 4D-MSPECT (Michigan University, USA), and sent to five institutions with different acquisition methods and software settings (Table 1) [1, 3]. The databases used in this study were based on Tc-99m MIBI and tetrofosmin with $360^{\circ}(\mathrm{n}=80)$ and $180^{\circ}(\mathrm{n}=56)$. Their ages, height, weight, heart rate and blood pressure at rest and exercise, ejection fraction, left ventricular volumes and regional distribution of the tracers were precisely described elsewhere [4-6]. The common database was not modified in each institution thereafter.

\section{Patients}

Two groups of patients were accumulated in five hospitals. Selection criteria for patients with coronary artery disease included (1) confirmed coronary artery stenosis $\geq 75 \%$ by coronary angiography (CAG), which was classified into single, double or triple-vessel diseases, (2) no history of old myocardial infarction and coronary revascularization, (3) no history of valvular heart disease, cardiomyopathy and severe arrhythmia, (4) no coronary intervention even if the patient underwent coronary angiography before, and (5) no changes of clinical signs and symptoms between SPECT and angiography. The near-normal patient group was defined as (1) no significant coronary artery stenosis by CAG or (2) no indications for CAG because of low-likelihood of coronary artery disease after screening of ischemic heart disease. In this near-normal group, additional criteria were no baseline cardiac disease, no medically treated complications of diabetes 
and hypertension, and no exercise ECG abnormality.

Based on these criteria, a total of 311 studies were accumulated, including single-vessel disease $(n=100)$, double-vessel disease $(n=44)$ and triple-vessel disease $(n=22)$ (Table 2). No patients with left main trunk stenosis were included. Since coronary angiography was performed in each institution, the method was not strictly regulated. However, the interpretation was performed by the cardiology specialists according to the criteria of segmentation and stenosis by American Heart Association. The average age was $68 \pm 11$ years (197 males, 114 females), and body surface area was $1.67 \pm 0.20 \mathrm{~m}^{2}$. Checked items in patients with low likelihood of coronary artery disease $(n=145)$ included no significant stenosis $(<70 \%)$ confirmed by CAG in $32 \%$, coronary artery disease denied by clinical work-ups in 51\%, no exercise ECG abnormality in $11 \%$ and no medically treated diabetes or hypertension in $49 \%$.

\section{SPECT data acquisition and software}

Table 1 summarizes the acquisition conditions and software types in five institutions. All institutions used multi-detector SPECT cameras. The rotation range was $180^{\circ}$ in two institutions and $360^{\circ}$ in three. The pixel size ranged from 6.4 to $6.9 \mathrm{~mm}$, and the camera rotation step was from $5.6^{\circ}$ to $6^{\circ}$ per projection. The software used for gated SPECT analysis was QPS with different versions and 4D-MSPECT $[1,3,7,8]$.

\section{Visual and quantitative analysis}

Visual analysis was performed in each institution by the nuclear cardiology specialist independent of quantitative data analysis. Existence of ischemia was determined by original myocardial stress-rest images and judged as positive or not without quantitative data. The visual interpretation was confirmed independently by two experts in the core laboratory. The consensus was based on $\geq 2$ of the 3 observers. A 17-segment model was applied in all institutions. Quantitative five-point scoring was performed as $0=$ normal, $1=$ slight decrease, $2=$ moderate decrease, $3=$ severe decrease and $4=$ complete defect. The scoring was performed automatically based on summed stress score (SSS), summed rest score (SRS) and summed difference score (SDS), in addition to ejection fraction (EF), end-diastolic volume (EDV) and end-systolic volume (ESV) at rest. Since old myocardial infarction was excluded in this study, we only used SSS at the stress condition for diagnosis of abnormality. For patient-based analysis, SSS $\geq 4$ of the whole segments was defined as the definite abnormality. For coronary artery-based analysis, the territory of the left anterior descending artery (LAD) was defined as anterior, anteroseptal and apical regions, that of the left circumflex artery (LCX) as anterolateral and inferolateral regions, and that of right coronary artery (RCA) as inferior and inferoseptal regions. Only regional scores $\geq 2$ of one principal culprit coronary artery territory was judged as abnormal. The principal culprit coronary artery was defined as the region of the severest stenotic artery even in patients with multi-vessel diseases.

\section{Statistics}

Mean and standard deviation was used for all parameters. Sensitivity and specificity were calculated in each hospital. However since one institution used only five patients with coronary stenosis and clinical background was variable among institutions, we mainly evaluated diagnostic ability of the total number of patients. The differences of the mean and variances were analyzed using statistics software JMP version 7 (SAS Institute, Inc. US and Canada Sales). $\mathrm{P}<0.05$ was considered significant. 


\section{Results}

Figure 2 shows an example patient with a three-vessel disease, involving $75 \%$ stenosis of the segment 1 (RCA, proximal), $75 \%$ stenosis of the segment 6 (LAD, proximal) and $90 \%$ stenosis of the segment 11 (LCX, proximal). Induced ischemia was judged in the LCX territory, and quantitative scoring was 16 points including LCX and RCA territories. In this case only the LCX territory was used for evaluating diagnostic ability, although quantitative analysis seemed to detect ischemia in the RCA territory. Table 2 summarizes the accumulated patient profiles. Mean EF was $63 \pm 13 \%$, and EDV and ESV were $89 \pm 41 \mathrm{ml}$ and $36 \pm 31 \mathrm{ml}$, respectively. The lowest EF and the highest EDV in the institution D depended on a higher inclusion rate of coronary artery disease.

In the patient-based analysis, sensitivity ranged from 0.40 to 0.80 by visual analysis and from 0.60 to 0.85 by quantitative analysis (Table 3 ). Specificity ranged from 0.72 to 0.95 by visual analysis and from 0.70 to 1.00 by quantitative analysis. Overall sensitivity, specificity and diagnostic accuracy were $0.72,0.79$ and 0.75 , respectively, by visual detection of ischemia $(\mathrm{p}=\mathrm{n}$. $\mathrm{s}$. for visual vs. quantitative analysis). Based on quantitative analysis with $\mathrm{SSS} \geq 4$ used for the abnormal stress study, overall sensitivity, specificity and diagnostic accuracy were $0.77,0.74$ and 0.75 , respectively. Coronary artery-based analysis demonstrated similar diagnostic performance of sensitivity, specificity and diagnostic accuracy of $0.71,0.79$ and 0.75 , respectively, by visual analysis, and those of $0.70,0.75$ and 0.73 by quantitative analysis, respectively $(p=n$. s.).

Regarding the lower specificity of quantitative analysis compared to that of visual analysis, we found 12 patients without $\mathrm{CAD}$ who showed abnormality in quantitative results, but were visually judged as normal. Of 12 patients, 11 were females, and 10 of these 11 had false positive score ( 1 or 2 for each segment) in the anterolateral regions, which was caused by breast attenuation (Figure 3). One male patient also showed an abnormal score in the anterior (score 2) and lateral (scores 2 and 1).

\section{Discussion}

The hypothesis of this study was that a single common database created by multiple institutions with similar acquisition parameters could be applied to any hospital under the terms of similar acquisition and processing. This study demonstrated comparable diagnostic ability between the institutional expert visual interpretation and the quantitative analysis using different software types and versions.

In ideal conditions, standard databases should be generated based on individual institutional conditions of the SPECT system and population. By comparing manufacturer databases and institutional databases, Knollmann et al. found some differences in quantitative scores between software algorithms as well as between manufacturer databases [9]. The merit of an individualized database is its specificity to camera types, camera rotation ranges, orbits, multi-detector configuration, and therefore higher diagnostic performance would be expected. The best-fitted databases to a specific study population might also be created separately. In contrast, the demerits of an institution-specific normal database involve not only the cumbersome procedures to make the databases, but also the use of different institutional normal databases in a multi-center study. In addition, normal databases adapted for each software and version should be created from different control subjects if the data could not been transferred successfully.

In a precedent study using the same normal database, we found that the American database and Japanese database demonstrated significant differences in scores, and the best diagnostic performance was observed by $180^{\circ} / 360^{\circ}$-arc rotation matched and nationality-matched databases [10]. In a Japanese population, although the JSNM database was generated by the compilation of multiple hospitals, application of the pre-installed American normal database supplied by the manufacturer degraded the diagnostic ability compared with that of the Japanese 
database.

The variability of sensitivity and specificity among institutions could be explained by the patient selection rather than by the diagnostic ability of each institute. In fact, some institutions examined relatively low-risk patient groups, and others had a number of CAG in the same period and a higher incidence of coronary artery disease. It should be noted that quantitative and visual analyses showed comparable values in all institutions. Slightly lower specificity for quantitative analysis was caused mainly by female breast attenuation. The breast attenuation artifact was judged correctly by visual analysis due to the non-coronary low count distribution. Moreover, attenuation artifacts can be judged by projection images and patient's breast size. Another option might be attenuation correction method, which was not used in this study. Comprehensive understanding is always required to avoid this sort of interpretation of artifacts.

The original JSNM working group database was prepared using a DICOM format of short-axis images, and it can be installed on any computer software. We have successfully installed the same normal databases into QPS with the latest and previous versions, 4D-MSPECT, Emory cardiac toolbox (Emory University, USA) and other currently developed software [1-3]. Since each software program has a different calculation algorithm, regional mean and deviation values on a polar map were not exactly identical. However, they were generated from the same sets of shortaxis images, and therefore have common background characteristics. Although we admit significant differences in regional counts among genders and camera rotation ranges [10-15], the institutional differences in the Japanese population seemed to be less critical if these factors were in agreement.

The principal culprit coronary artery was evaluated for detection of ischemia, although additional ischemia could be detected as shown in Figure 2. Even in multi-vessel disease, the induced ischemia may not be detected in all of the stenotic artery territories $[16,17]$. Myocardial perfusion imaging has rather been used to identify the culprit lesion for ischemia in multiple stenotic lesions. To detect multi-vessel high- risk patients, additional information, such as multiple defects $>1$ coronary supply region, transient ischemic dilatation after stress, multiple areas of abnormal regional wall motion, increased volume and high lung uptake, might have incremental values.

This study has some limitations. Visual analysis was performed only to judge positive and negative ischemia in each coronary territory. Since visual scoring criteria of each physician could not be completely unified, we accepted ordinary decision criteria of each institution, and visual analysis was compared with the institutional quantitative analysis based on JSNM standards. For the $180^{\circ}$ rotation method, the rotation orbit included both circular and non-circular orbits, and the camera configuration was not specific to $90^{\circ}$ and $79^{\circ}$ rotations. In addition, the $180^{\circ}$ camera setting included various preferences for acquisition parameters including centering of heart and a variable camera-heart distance according to the orbit. Since specific databases that included all these factors would increase the number of database types, the JSNM working group decided to simplify the database type to only include the combinations of radiopharmaceuticals $\left({ }^{99 \mathrm{~m}} \mathrm{Tc}\right.$ perfusion tracers, ${ }^{201} \mathrm{Tl},{ }^{123} \mathrm{I} \mathrm{BMIPP}$ and $\left.{ }^{123} \mathrm{I} \mathrm{MIBG}\right)$, rotation range $\left(180^{\circ}\right.$ and $360^{\circ}$ rotation acquisitions $)$ and genders. The software was not the latest version in some institutes including QPS and 4D-MSPECT, although we understood that the algorithm had been updated by the software developers. Therefore, better diagnostic yields might be expected by the unified computer algorithm with the newest versions.

In conclusion, a common database created by the JSNM working group activity provided comparable diagnostic performance to expert interpretation of the institutes despite variations of acquisitions and software programs. Since the precision of QGS results was generally good, even if the same data were separately processed in each institution [18], a common normal database might be used as part of a multi-center study. 


\section{Acknowledgment}

This work was performed as part of the JSNM working group activity and supported by Grants-inAid for Scientific Research in Japan (PI: Kenichi Nakajima). We also thank GE Yokogawa Medical Systems for providing a workstation for data processing. The research version of QPS software was supplied by Drs Germano and Slomka (Cedars Sinai Medical Center, USA). 
Table 1. Data acquisition system and data processing

\begin{tabular}{llllll}
\hline Institutions & $\mathrm{A}$ & $\mathrm{B}$ & $\mathrm{C}$ & $\mathrm{D}$ & $\mathrm{E}$ \\
\hline Camera vendor & GE & Toshiba & Siemens & GE & $\begin{array}{l}\text { Toshiba/ } \\
\text { Siemens }\end{array}$ \\
\hline $\begin{array}{l}\text { Number of detectors } \\
\text { Detector configuration }\left(^{\circ}\right)\end{array}$ & 2 & 3 & 2 & 2 & 2 \\
\hline $\begin{array}{l}\text { Acquisition parameters } \\
\text { Rotation range }\left(^{\circ}\right)\end{array}$ & 180 & 360 & 180 & 360 & 360 \\
\hline $\begin{array}{l}\text { Orbit } \\
\text { triangle }\end{array}$ & 90 & 180 & 180 \\
\hline $\begin{array}{l}\text { Pixel size }(\mathrm{mm}) \\
\text { Step }\left(^{\circ}\right)\end{array}$ & Circular & Circular & $\begin{array}{l}\text { Non- } \\
\text { circular }\end{array}$ & Circular & Circular \\
\hline $\begin{array}{l}\text { Quantification software } \\
\text { Software type }\end{array}$ & 5.8 & 6.4 & 6.6 & 6.9 & 6.6 \\
\hline Version & QPS & $\begin{array}{l}\text { 4D- } \\
\text { MSPECT }\end{array}$ & QPS & QPS & QPS \\
\hline
\end{tabular}

Table 2. Patient characteristics in five institutions

\begin{tabular}{|c|c|c|c|c|c|c|}
\hline Institutions & A & $\mathrm{B}$ & $\mathrm{C}$ & $\mathrm{D}$ & $\mathrm{E}$ & Total \\
\hline Number of patients & 19 & 40 & 40 & 172 & 40 & 311 \\
\hline Age (years) & $66 \pm 10$ & $73 \pm 9$ & $68 \pm 10$ & $67 \pm 11$ & $68 \pm 13$ & \\
\hline Male/Female & $8 / 11$ & $25 / 15$ & $30 / 10$ & $107 / 65$ & $27 / 13$ & $\begin{array}{l}197 \\
114\end{array}$ \\
\hline $\operatorname{BSA}\left(\mathrm{m}^{2}\right)$ & $\begin{array}{l}1.57 \\
\pm 0.17\end{array}$ & $1.63 \pm 0.21$ & $1_{ \pm 0.17}^{1}$ & $3_{1.66 \pm 0.20}$ & $01.70 \pm 0.25$ & \\
\hline \multicolumn{7}{|l|}{ Coronary stenosis } \\
\hline $\begin{array}{l}\text { No stenosis or low- } \\
\text { likelihood }\end{array}$ & -14 & 20 & 20 & 71 & 20 & 145 \\
\hline SVD & 5 & 15 & 16 & 53 & 11 & 100 \\
\hline DVD & 0 & 3 & 2 & 32 & 7 & 44 \\
\hline TVD & 0 & 2 & 2 & 16 & 2 & 22 \\
\hline \multicolumn{7}{|l|}{ LV function } \\
\hline $\mathrm{EF}(\%)$ & $69 \pm 17$ & $64 \pm 9$ & $64 \pm 18$ & $59 \pm 10$ & $67 \pm 10$ & \\
\hline $\mathrm{EDV}(\mathrm{ml})$ & $74 \pm 36$ & $75 \pm 22$ & $87 \pm 61$ & $109 \pm 35$ & $75 \pm 25$ & \\
\hline ESV (ml) & $29 \pm 30$ & $28 \pm 14$ & $41 \pm 54$ & $45 \pm 23$ & $26 \pm 16$ & \\
\hline
\end{tabular}

Abbreviations

CAD, coronary artery disease; SVD, single-vessel disease; DVD, double-vessel disease; TVD, triple-vessel disease; LV, left ventricle; EF, ejection fraction; EDV, end-diastolic volume; ESV, end-systolic volume 
Table 3. Diagnostic accuracy by visual and quantitative analyses

\begin{tabular}{|c|c|c|c|c|c|c|}
\hline \multirow[b]{2}{*}{ Institution } & \multicolumn{3}{|l|}{ Visual } & \multicolumn{3}{|l|}{ Quantitative } \\
\hline & Sensitivity & Specificity & Accuracy & Sensitivity & Specificity & Accuracy \\
\hline \multicolumn{7}{|c|}{ Patient-based } \\
\hline A & 0.40 & 0.86 & 0.74 & 0.60 & 0.93 & 0.84 \\
\hline B & 0.80 & 0.95 & 0.88 & 0.75 & 1.00 & 0.88 \\
\hline $\mathrm{C}$ & 0.75 & 0.80 & 0.78 & 0.85 & 0.80 & 0.83 \\
\hline $\mathrm{D}$ & 0.70 & 0.72 & 0.71 & 0.76 & 0.62 & 0.70 \\
\hline E & 0.75 & 0.80 & 0.78 & 0.75 & 0.70 & 0.73 \\
\hline Total & 0.72 & 0.79 & 0.75 & 0.77 & 0.74 & 0.75 \\
\hline Number & $119 / 166$ & $114 / 145$ & $233 / 311$ & $127 / 166$ & $107 / 145$ & $234 / 311$ \\
\hline \multicolumn{7}{|c|}{ Coronary artery-based } \\
\hline A & 0.40 & 0.86 & 0.74 & 0.40 & 0.93 & 0.79 \\
\hline $\mathrm{B}$ & 0.80 & 0.95 & 0.88 & 0.75 & 1.00 & 0.88 \\
\hline $\mathrm{C}$ & 0.70 & 0.80 & 0.75 & 0.80 & 0.85 & 0.83 \\
\hline D & 0.70 & 0.72 & 0.71 & 0.71 & 0.63 & 0.68 \\
\hline E & 0.75 & 0.80 & 0.78 & 0.60 & 0.70 & 0.65 \\
\hline Total & 0.71 & 0.79 & 0.75 & 0.70 & 0.75 & 0.73 \\
\hline Number & $118 / 166$ & $114 / 145$ & $232 / 311$ & $117 / 166$ & $109 / 145$ & $226 / 311$ \\
\hline
\end{tabular}

Number of patients with coronary artery disease in 5 institution was 5 of 19 for A, 20 of 40 for B, 20 of 40 for C 101 of 172 for D and 20 of 40 for E. In the coronary artery-based analysis, only principal culprit coronary artery was analyzed for detection of ischemia. 

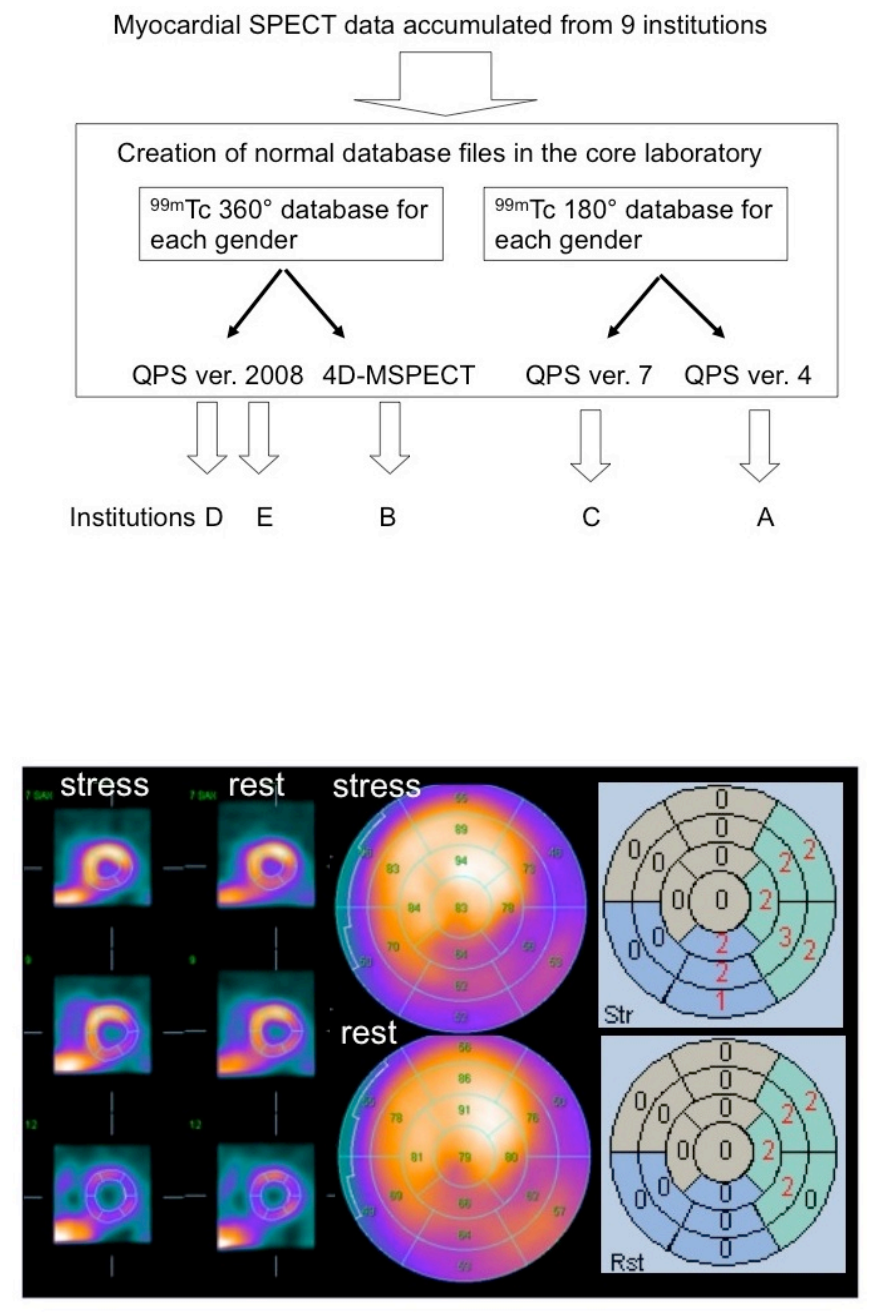

Figure 1. Normal database accumulation by JSNM working group and distribution of the databases to each institute.
Figure 2.

A patient with three-vessel disease. Hypoperfusion areas were observed in the lateral to inferolateral regions and ischemia was positively diagnosed. The culprit lesion was judged in the LCX territory both by visual and quantitative analyses.

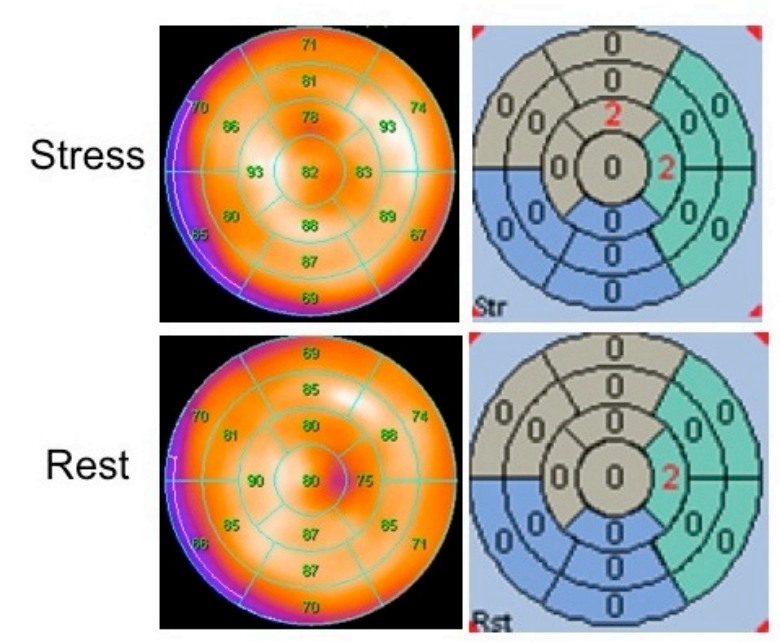

\section{Figure 3}

Polar maps from a female patient showing anterior and lateral scores. By visual analysis, the small regional decrease was explained by breast attenuation and its movement at resting map. Based on quantitative scores, small segmental scores were calculated. 


\section{References}

1. Germano G, Kavanagh PB, Slomka PJ, Van Kriekinge SD, Pollard G, Berman DS. Quantitation in gated perfusion SPECT imaging: the Cedars-Sinai approach. J Nucl Cardiol. 2007;14:433-54.

2. Garcia EV, Faber TL, Cooke CD, Folks RD, Chen J, Santana C. The increasing role of quantification in clinical nuclear cardiology: the Emory approach. J Nucl Cardiol. 2007;14:420-32.

3. Ficaro EP, Lee BC, Kritzman JN, Corbett JR. Corridor4DM: the Michigan method for quantitative nuclear cardiology. J Nucl Cardiol. 2007;14:455-65.

4. Nakajima K, Kumita S, Ishida Y, Momose M, Hashimoto J, Morita K, et al. Creation and characterization of Japanese standards for myocardial perfusion SPECT: database from the Japanese Society of Nuclear Medicine Working Group. Ann Nucl Med. 2007;21:505-11.

5. Matsuo S, Nakajima K, Yamashina S, Sakata K, Momose M, Hashimoto J, et al. Characterization of Japanese standards for myocardial sympathetic and metabolic imaging in comparison with perfusion imaging. Ann Nucl Med. 2009;23:517-22.

6. Akhter N, Nakajima K, Okuda K, Matsuo S, Yoneyama T, Taki J, et al. Regional wall thickening in gated myocardial perfusion SPECT in a Japanese population: effect of sex, radiotracer, rotation angles and frame rates. Eur J Nucl Med Mol Imaging. 2008;35:1608-15.

7. Slomka PJ, Nishina H, Berman DS, Akincioglu C, Abidov A, Friedman JD, et al. Automated quantification of myocardial perfusion SPECT using simplified normal limits. J Nucl Cardiol. 2005; 12:66-77.

8. Slomka PJ, Nishina H, Berman DS, Kang X, Friedman JD, Hayes SW, et al. Automatic quantification of myocardial perfusion stress-rest change: a new measure of ischemia. J Nucl Med. 2004;45:183-91.

9. Knollmann D, Knebel I, Koch KC, Gebhard M, Krohn T, Buell U, et al. Comparison of SSS and SRS calculated from normal databases provided by QPS and 4D-MSPECT manufacturers and from identical institutional normals. Eur J Nucl Med Mol Imaging. 2008;35:311-8.

10. Nakajima K, Okuda K, Kawano M, Matsuo S, Slomka P, Germano G, et al. The importance of population-specific normal database for quantification of myocardial ischemia: comparison between Japanese 360 and 180-degree databases and a US database. J Nucl Cardiol. 2009;16:422-30.

11. Eisner RL, Nowak DJ, Pettigrew R, Fajman W. Fundamentals of 180 degree acquisition and reconstruction in SPECT imaging. J Nucl Med. 1986;27:1717-28.

12. Van Train KF, Areeda J, Garcia EV, Cooke CD, Maddahi J, Kiat H, et al. Quantitative same-day reststress technetium-99m-sestamibi SPECT: definition and validation of stress normal limits and criteria for abnormality. J Nucl Med. 1993;34:1494-502.

13. Bice AN, Clausen M, Loncaric S, Wagner HN, Jr. Comparison of transaxial resolution in 180 degrees and 360 degrees SPECT with a rotating scintillation camera. Eur J Nucl Med. 1987;13:7-11.

14. Tamaki N, Mukai T, Ishii Y, Fujita T, Yamamoto K, Minato K, et al. Comparative study of thallium emission myocardial tomography with 180 degrees and 360 degrees data collection. J Nucl Med. 1982;23:661-6.

15. Go RT, MacIntyre WJ, Houser TS, Pantoja M, O'Donnell JK, Feiglin DH, et al. Clinical evaluation of 360 degrees and 180 degrees data sampling techniques for transaxial SPECT thallium-201 myocardial perfusion imaging. J Nucl Med. 1985;26:695-706.

16. Elhendy A, Sozzi FB, van Domburg RT, Bax JJ, Geleijnse ML, Valkema R, et al. Accuracy of exercise stress technetium $99 \mathrm{~m}$ sestamibi SPECT imaging in the evaluation of the extent and location of coronary artery disease in patients with an earlier myocardial infarction. J Nucl Cardiol. 2000;7:432-8.

17. Forster S, Rieber J, Ubleis C, Weiss M, Bartenstein P, Cumming P, et al. Tc-99m sestamibi single photon emission computed tomography for guiding percutaneous coronary intervention in patients with multivessel disease: a comparison with quantitative coronary angiography and fractional flow reserve. Int $\mathbf{J}$ Cardiovasc Imaging. 2009.

18. Nakajima K, Nishimura T. Inter-institution preference-based variability of ejection fraction and volumes using quantitative gated SPECT with $(99 \mathrm{~m}) \mathrm{Tc}$-tetrofosmin: a multicentre study involving 106 hospitals. Eur J Nucl Med Mol Imaging. 2006;33:127-33. 